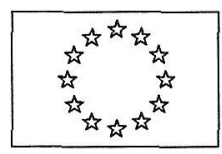

COMISIÓN DE LAS COMUNIDADES EUROPEAS

Bruselas, 30.11.2006 COM (2006) 735 final

COMUNICACIÓN DE LA COMISIÓN

AL CONSEJO Y AL PARLAMENTO EUROPEO

\title{
EL PLANTEAMIENTO GLOBAL SOBRE LA MIGRACIÓN UN AÑO DESPUÉS: HACIA UNA POLÍTICA GLOBAL EUROPEA EN MATERIA DE MIGRACIÓN
}

\section{RESPUESTA A LOS DESAFÍOS DE LA MIGRACIÓN}

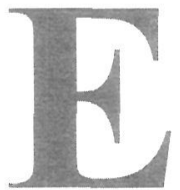

n octubre de 2005, los Jefes de de Estado y de Gobierno trataron sobre la migración como uno de los principales desafíos de la globalización y acordaron la urgente necesidad de intensificar la acción en este ámbito. La UE supo reaccionar con rapidez. En el plazo de un mes, la Comisión presentó un Plan para un amplio programa de acción, Acciones prioritarias para hacer frente a los desafios de la migración: Primera etapa en el proceso de seguimiento de Hampton Court ${ }^{1}$. Esta Comunicación recogía ya una serie de medidas concretas que constituían un planteamiento global sobre la migración y fueron la base para debates ulteriores en el seno del Consejo Europeo sobre África y la región mediterránea.

En diciembre de 2005, el Consejo Europeo adoptó El planteamiento global sobre migración: Acciones prioritarias en África y el Mediterráneo. Este enfoque global define una acción y políticas coherentes en materia de migraciones, se ocupa de un extenso abanico de problemas vinculados a la migración y cubre múltiples ámbitos de acción al respecto: relaciones exteriores, desarrollo y empleo, y justicia, libertad y seguridad. El enfoque adoptado muestra una solidaridad real y activa basada en una división de responsabilidades entre los Estados miembros y los terceros países.

El fenómeno migratorio ha existido en todas las épocas de la historia. A escala planetaria, el número de emigrantes en porcentaje de la población mundial no es hoy más elevado que en otros períodos históricos. No obstante, las mi-

1 Las referencias a todos los documentos citados están enumeradas en el anexo. 
graciones han adquirido una mayor importancia para Europa. Ésta ha pasado en efecto en algunas décadas de ser una región de emigración a ser una de las grandes regiones de destino. Según la OCDE, los principales países de origen de los flujos de migración hacia la UE en 2004 eran Rumania, Marruecos, Bulgaria, Turquía, Ucrania, y la Federación Rusa. Los flujos de inmigración se han diversificado más, con un número creciente de inmigrantes procedentes de Europa Central y Oriental, Asia (en particular China), y América Central y Latina (especialmente Ecuador). La migración procedente de África ha crecido sustancialmente en los últimos meses. Es improbable que este fenómeno se detenga en un futuro próximo y las presiones migratorias podrían incluso intensificarse. Al mismo tiempo, habida cuenta de su evolución demográfica, la UE necesitará inmigrantes para garantizar la viabilidad de sus mercados laborales. La UE debe hacer frente a la competencia de las otras regiones del mundo y necesita inmigrantes dotados con las cualificaciones necesarias para ello.

La presente Comunicación tiene dos objetivos. Primero, responder a la invitación del Consejo Europeo a la Comisión para que informe sobre los progresos alcanzados en la ejecución de la primera fase del Planteamiento global y las acciones prioritarias previstas para África y el Mediterráneo para finales de 2006. En segundo lugar, propone vías para que el planteamiento de la Unión Europea sea verdaderamente global. Esto responde a las llamadas hechas por los Ministros de Justicia e Interior en Tampere el 21 de septiembre y los Jefes de Estado y de Gobierno en Lahti el 20 de octubre para intensificar los esfuerzos. Por lo tanto, sugiere incluir otros ámbitos políticos que no formaban parte del Planteamiento global de diciembre de 2005, tales como medidas legales en materia de migración e integración. De esta manera, la colaboración con terceros países abordará una gama completa de aspectos de interés y de preocupación para todas las partes implicadas.

En este marco, dada la necesidad urgente de consolidar las políticas de la UE en materia de migración y los ámbitos relacionados con ella, debería aumentarse la eficacia de los procedimientos de toma de decisiones, también en el campo de la migración legal, puesto que permitiría a la Unión responder a las expectativas de los ciudadanos en este ámbito. El Tratado por el que se instituye una Constitución para Europa ofrece la solución apropiada a las carencias actuales en el proceso de toma de decisiones, aplicando a la migración legal las normas de la toma de decisiones por mayoría cualificada y del procedimiento legislativo ordinario (codecisión). En la actual situación, la "cláusula pasarela" contenida en el artículo 67 TCE ofrecería una herramienta importante para lograr este objetivo, paralelamente a la búsqueda de un Acuerdo institucional completo en el marco creado por el Consejo Europeo de junio de 2006, y debe por ello aplicarse.

En un esfuerzo por consolidar más la política global de migración de la UE, la Comisión ha creado un grupo de Comisarios sobre migración. Un empeño similar con el fin de reunir ámbitos políticos diversos aunque relacionados entre sí, se aprecia en varios Estados miembros y se debe acoger con satisfacción esta tendencia y, cuando sea posible, fomentarla.

Aunque la presente Comunicación se centra en África y la región del Mediterráneo, el prurito de globalidad también debe traducirse en el futuro 
en términos geográficos. Debería prestarse mayor atención a la aplicación del planteamiento a otras regiones, en especial aquéllas en las fronteras externas del este y del sudeste de la UE a la vista de los desafíos que plantean estas rutas migratorias. Además, aunque no sea una prioridad a corto plazo, debe tenerse en cuenta la importancia cada vez mayor de los problemas relacionados con la migración en nuestras relaciones con los países asiáticos y latinoamericanos y las expectativas cada vez mayores de estos países en materia de cooperación con la UE. Además, la reciente Cumbre iberoamericana reclamó la intensificación del diálogo y la cooperación en asuntos de migración y dar una expresión concreta, en las políticas nacionales, en los foros subregionales y los Acuerdos internacionales, al compromiso de Montevideo sobre migración y desarrollo. De hecho, muchas de las medidas y políticas descritas en el apartado 3 podrían aplicarse, de una manera similar, a nuestra cooperación y diálogo con América Latina y Asia.

\section{APLICACIÓN DEL PLANTEAMIENTO GLOBAL: RESULTADOS DEL PRIMER AÑO EN ÁFRICA Y LA REGIÓN DEL MEDITERRÁNEO}

El año 2006 se consagró a establecer un programa de trabajo con África. El pasado mes de julio se celebró en Rabat una Conferencia ministerial sobre migración y desarrollo que reunió a unos 60 países ubicados a lo largo de las rutas migratorias de África central y occidental. Los Estados africanos y los de la UE participaron en septiembre en el diálogo de alto nivel de las Naciones Unidas sobre migraciones y desarrollo. En noviembre se celebró en Libia una Conferencia Ministerial UE-África sobre migración y desarrollo con el fin de formular por primera vez un enfoque de las migraciones de común acuerdo entre la UE y el conjunto de África. La cuestión de las migraciones ha figurado repetidamente en el orden del día de los programas de diálogo y cooperación con los países mediterráneos, elaborados sobre la base de los considerables trabajos llevados ya a cabo en el marco de la Política Europea de Vecindad (PEV), y el Foro Euromed ha sido utilizado para promover el intercambio de buenas prácticas y trabajar en un programa de actuación común. También figuró en el orden del día de las reuniones de alto nivel celebradas con la Unión Africana y las organizaciones regionales. Asimismo, se inició un diálogo sobre las cuestiones relativas a las migraciones con los principales Estados de África subsahariana sobre la base del artículo 13 del Acuerdo de Cotonú y se utilizó el mecanismo de reacción rápida para apoyar a Mauritania y Senegal en sus esfuerzos para luchar contra las migraciones ilegales.

En menos de doce meses, ya se han iniciado los trabajos de todas las acciones prioritarias. Para finales de año, FRONTEX habrá puesto en marcha y coordinado varias operaciones marítimas conjuntas en las regiones atlántica y mediterránea, concluido un análisis de riesgo para África y presentado estudios de viabilidad sobre el establecimiento de una red mediterránea de patrullas costeras y un sistema de vigilancia que cubra por entero la frontera marítima meridional de la UE y del Mar Mediterráneo. Se están creando redes regionales de funcionarios de enlaceresponsables de inmigración (ILO) en las 
rutas clave de migración a través de África. La Comisión ha publicado, por otra parte, una propuesta de creación de equipos de intervención rápida en las fronteras y ha llevado a cabo un análisis del Derecho internacional del Mar.

Un informe más detallado sobre la aplicación del planteamiento global se recoge en el anexo $B$.

\section{REFORZAR LA COHERENCIA: UNA POLÍTICA DE MIGRACIÓN EUROPEA GLOBAL}

El enfoque global debe asentarse firmemente sobre tres principios: solidaridad entre los Estados miembros, asociación con los terceros países y protección de los emigrantes, particularmente los grupos más vulnerables tales como los menores no acompañados y mujeres. Los tres apartados siguientes sugieren los medios para desarrollar este enfoque tanto con África como con los otros socios.

3.1. Consolidar el diálogo y la cooperación con los paises africanos de origen y de tránsito

\subsubsection{Diálogo con los paises africanos}

El marco global para la cooperación de la UE con África viene definido por la Estrategia de la UE para África, adoptada por el Consejo Europeo de los días 15 y 16 de diciembre de 2005. Los principales propósitos de esta estrategia son la realización de los objetivos de desarrollo del milenio y la promoción del desarrollo sostenible, la seguridad y la buena gobernanza en África. El trabajo futuro en el ámbito de la migración debe ser coherente con esta estrategia, especialmente en relación con la conveniencia de abordar las causas profundas de la migración tales como la pobreza, los conflictos y el desempleo. Otras políticas de la UE, incluidas el comercio, la agricultura y la pesca, deberían servir a los mismos objetivos a través de una mayor coherencia de las políticas para el desarrollo (CPD).

La Conferencia ministerial UE-África sobre migración y desarrollo celebrada en Libia los días 22 y 23 de noviembre de 2006 identificó con éxito diversas prioridades para la cooperación y adoptó una declaración ambiciosa que prepara el camino para la acción conjunta entre África y la UE a nivel continental, regional y nacional en diversas áreas, en torno a la lucha contra la migración ilegal, la facilitación de los desplazamientos regulares de las personas y la acción para abordar las causas profundas de la migración. África y la UE deberían comprometerse seriamente en la ejecución de las acciones recogidas en la Declaración final para lograr unos resultados concretos con el fin de informar a la segunda Conferencia ministerial que se celebrará en el plazo de tres años.

A nivel regional, la conferencia de Rabat celebrada en julio fue también un éxito y preparó el camino para una acción adaptada a unas rutas migratorias específicas. Los esfuerzos deberían concentrarse en garantizar un seguimiento apropiado con objeto de evaluar los avances realizados en 
una segunda Conferencia ministerial dentro de dos años. Los contactos entre la UE y los Estados de África oriental deberían también promoverse más con el fin de desarrollar la cooperación concreta a lo largo de las rutas de la migración de África oriental.

Sobre la base del trabajo llevado a cabo sobre las rutas migratorias, la UE estimulará una cooperación más específica con diversas regiones en África. Las plataformas de cooperación reunirán a los países africanos, los Estados miembros de la UE y las organizaciones internacionales en un esfuerzo para gestionar la migración más eficazmente en interés de todos. Este marco común podría entonces permitir la formulación de acuerdos regionales con los países africanos interesados.

En el marco de la Política Europea de Vecindad (PEV), se desarrollarán más el diálogo y la cooperación con los países africanos del norte a nivel bilateral y regional. Los problemas de la migración constituyen un elemento esencial en nuestros Planes de Acción de la PEV, nuestro diálogo político y la ayuda financiera prestada para consolidar las capacidades de estos países para gestionar la migración más eficazmente. Deben continuarse asimismo los trabajos en el contexto de EUROMED. Los socios han aceptado dar un seguimiento concreto al capítulo relativo a la migración, la integración social, la justicia y la seguridad del programa de trabajo quinquenal acordado en Barcelona en noviembre de 2005. Esto debería dar paso a una Conferencia ministerial sobre migración que debe convocarse en la segunda mitad de 2007.

La UE también continuará integrando los problemas de migración en el diálogo político periódico con todos los países ACP y las Comunidades económicas regionales (CER). Este diálogo está basado en el amplio programa del artículo 13 del Acuerdo de Cotonú, que cubre una gama variada de temas en el campo de la migración y el desarrollo. Por parte de la UE, serán las Delegaciones de la Comisión y las embajadas de la Presidencia en los países concernidos las que se encarguen del diálogo conforme a lo previsto en el artículo 8 de Cotonú. En su caso, el diálogo se reforzará a través de misiones específicas de la Comisión. En 2006 se llevaron a cabo tres de estas "misiones del artículo 13". También las recibirán otros países africanos clave en 2007: Camerún, Etiopía, Ghana y Nigeria. En ese mismo año se reunirá por primera vez un grupo de trabajo conjunto CEDEAO-UE sobre migración.

La UE debería también tener un papel activo en el seguimiento del diálogo de alto nivel de la ONU sobre migración y desarrollo, en especial al constituirse el foro global sobre migración. La Comisión está dispuesta y preparada para contribuir activamente a la primera reunión de este foro que se celebrará en Bélgica en el verano de 2007.

\subsubsection{Promover el Programa sobre migración y desarrollo}

Por lo que respecta al Programa sobre migración y desarrollo, el primer desafío es abordar los principales factores que alientan la migración: la pobreza y la falta de oportunidades de trabajo. La UE debe reconocer que la creación de puestos de trabajos en los países menos desarrollados puede 
reducir perceptiblemente la presión migratoria que parte de África. Los emigrantes deberían contar con apoyo si deciden contribuir al desarrollo de sus países de origen. Además, las remesas de emigrantes continuarán aumentando, y la Comisión está explorando mecanismos de cooperación en este campo con instituciones tales como el Banco Mundial y el Banco Europeo de Inversiones. Los esfuerzos se concentrarán en la mejora del acopio de datos, la reducción de los costes de transferencia, la facilitación del uso del sector financiero como canal de transferencia y la exploración de vías con objeto de aumentar el impacto de las remesas en las políticas de desarrollo.

El programa de la UE sobre migración y desarrollo en África intentará proporcionar una respuesta experimental a corto plazo al desafío de la migración para los países ACP. EI primer objetivo de esta iniciativa de programación conjunta sería abordar de manera seria la falta de puestos de trabajo decentes en África. Promover inversiones en sectores que demandan grandes cantidades de mano de obra en las regiones con altas tasas de migración exterior será una gran prioridad en un contexto más amplio destinado a facilitar la migración y la movilidad laboral entre los países africanos. Otras áreas de cooperación incluirán las remesas, la fuga de cerebros, las diásporas, la buena gobernanza y la migración ilegal y la trata de seres humanos. Se invitará a los Estados miembros a trabajar conjuntamente con la Comisión en la programación de estas acciones. La Comisión reservará una cantidad de 40 millones de euros para la primera fase de esta iniciativa (recursos $9^{\circ} \mathrm{FED}$ ), que será complementada por las contribuciones de los Estados miembros. El alcance geográfico de la iniciativa será el África subsahariana, pero las acciones pueden centrarse en países o regiones específicos y -en su primera fase- especialmente en África oriental.

La UE debe continuar ayudando a los Estados africanos a construir su capacidad para gestionar la migración y el asilo, incluidos los flujos SurSur, sin perjuicio de los Acuerdos regionales existentes sobre la migración y la movilidad. Se están tomando iniciativas importantes en países de la PEV en relación con el refuerzo de capacidades, en especial por lo que se refiere a la gestión aduanera y el apoyo institucional con el fin de mejorar la acogida de emigrantes así como la protección de los derechos de los emigrantes. Además, la Comisión ha propuesto crear unos perfiles de migración para cada país en vías de desarrollo interesado ${ }^{2}$. Los perfiles de la migración son una herramienta política que sirve para recopilar y analizar la información pertinente necesaria para desarrollar medidas concretas en una situación dada en el ámbito de la migración y el desarrollo. Se están elaborando perfiles de la migración resumidos para todos los paises ACP como anexo a la nueva generación de documentos estratégicos nacionales. Los perfiles de la migración deberían establecerse para todos los países socios a medio plazo. Esta información puede posteriormente utilizarse para proporcionar ayuda técnica y financiera a los países africanos para solucionar las causas profundas de los flujos de migración. Una idea podría ser crear equipos de apoyo a la migración (EAM) integrados por expertos de 
los Estados miembros de la UE que podrían proporcionar la ayuda necesaria a los Estados africanos que lo soliciten. Europa debería mostrarse más activa y previsora por lo que se refiere a las cuestiones de la migración.

Las políticas de migración deben basarse en datos fiables y ser coherentes con otras políticas relacionadas. Promover los vínculos entre política e investigación contribuiría a una mejor comprensión de las realidades de la migración y a la elaboración de las políticas en la materia. Inspirada por la red académica que liga a los Institutos de investigación sobre migración en los países mediterráneos (CARIM), la Comisión apoyará iniciativas que estimulen el establecimiento de una Red panafricana de 'observatorios' sobre migración e institutos de investigación sobre el fenómeno de la migración.

Deberían también ponerse en marcha medidas de hermanamiento utilizando la financiación de la CE para ayudar a los países africanos a desarrollar sus políticas de migración y asilo. Además, las delegaciones de la Comisión y las misiones de los Estados miembros en los países africanos deberían garantizar que pueden tratar los problemas relacionados con la migración a través de la designación de una persona de contacto para als cuestiones de migración.

\subsection{Migración legal}

El desarrollo futuro de una política europea común de inmigración laboral es un componente importante del Planteamiento global. Para una política de migración europea verdaderamente global, la migración legal debe integrarse en las políticas exteriores e internas de la UE. La migración puede contribuir a cubrir las necesidades cambiantes del mercado laboral y debe tener en cuenta los aspectos económicos de la política de migración. El 28 de noviembre, el Consejo de Economía y Finanzas adoptó varias conclusiones sobre las diferentes políticas con el fin de aumentar los beneficios económicos que supone la migración para la UE. Tal como se recoge en el Plan político sobre migración legal, y conforme a los objetivos de la estrategia europea de empleo, la UE está adoptando un planteamiento de dos vías para los próximos años: facilitar la admisión de ciertas categorías de inmigrantes en un planteamiento basado en las necesidades existentes (por ejemplo, los trabajadores temporeros y trabajadores con un alto nivel de formación) sin perjuicio de la aplicación del principio de preferencia comunitario y proporcionar un estatus jurídico seguro común a todos los trabajadores inmigrantes legales.

Otras acciones deberían facilitar la adaptación entre la demanda y la oferta de trabajo. El futuro Portal de Inmigración, el portal europeo de la movilidad en el trabajo, las nuevas directrices 2007-2010 para la red EURES y el portal europeo para la movilidad de los investigadores son todas ellas herramientas fundamentales para lograr este objetivo. Se proporcionará a los terceros países información sobre las posibilidades legales de trabajar en Europa, incluyendo campañas de información específicas. Estimulando la formación profesional, los programas de desarrollo de las capacidades y los cursos de idiomas, los emigrantes potenciales podrán aumentar sus posibilidades de encontrar un empleo legal. Con este fin, podrían crearse centros específicos de migración en 
los países socios, apoyados por la financiación de la CE. Tales centros podrían también desempeñar un papel en la facilitación de la gestión de los temporeros, los intercambios de estudiantes e investigadores y otras formas de desplazamiento legal de las personas.

Paralelamente, debería explorarse más el potencial ofrecido por las nuevas formas de migración, en especial la migración circular. Además, será importante apoyar el desarrollo de herramientas para mejorar la gestión de la migración laboral que llega la UE desde terceros países. Esto requerirá el apoyo a actividades tales como el refuerzo de los servicios administrativos de los terceros países responsables de la gestión laboral de la migración; el desarrollo de las capacidades de los servicios de empleo nacionales en terceros países y la creación de centros de mediación así como la aplicación de planes de preinmigración en los países de origen.

Una vez que se han cumplido ciertas condiciones, por ejemplo, la cooperación en materia de migración ilegal y la celebración de acuerdos de readmisión, el objetivo podría ser acordar paquetes de movilidad con varios terceros países interesados que permitieran que sus ciudadanos tuvieran mejor acceso a la UE. Existe una clara necesidad de organizar mejor las diversas formas de desplazamiento legal entre la UE y los terceros países. Los paquetes de movilidad proporcionarían el marco global para gestionar tales movimientos y reunirían las posibilidades ofrecidas por los Estados miembros y la Comunidad Europea, al mismo tiempo que se respeta rigurosamente la división de competencias según lo previsto por el Tratado.

En el marco de paquetes más amplios relativos al establecimiento de Acuerdos de readmisión, la consolidación de la cooperación en materia de inmigración ilegal y el trabajo conjunto para una gestión aduanera efectiva pueden ser todos ellos requisitos previos para facilitar el visado. La Comisión considera que la facilitación de la movilidad es un elemento importante en el marco de un Planteamiento global de la migración, particularmente para los países cubiertos por la PEV en los que se requerirá un examen riguroso sobre la manera en que los procedimientos de visado podrían suponer menos un obstáculo para los viajes legales a la UE, y viceversa ${ }^{3}$.

Para desarrollar las políticas que tienen en cuenta los beneficios potenciales para los terceros países sobre la migración laboral hacia Europa, la UE debe ser muy consciente de los riesgos de fuga de cerebros y de sus consecuencias socioeconómicas para los países en vías de desarrollo. Por ejemplo, el reconocimiento de los efectos negativos de la emigración excesiva de trabajadores expertos en el servicio sanitario de varios países ha llevado al desarrollo de una estrategia coordinada de la UE, que incluye elaborar un conjunto de principios que rigen la contratación ética de profesionales sanitarios y la promoción de una mayor autosuficiencia en la formación de trabajadores en el ámbito de la salud en Europa para minimizar la demanda futura por parte de unos sistemas sanitarios poco asentados. Deberian emprenderse igualmente iniciativas para hacer frente a las carencias (potenciales) de cualificación técnica y la fuga de

3 Véase la Comunicación "Refuerzo de la PEV" COM (2006) 726 final. 
cerebros en otros sectores. Los beneficios relacionados con la circulación de cerebros deben ser estudiados con detenimiento en este contexto.

\subsection{Integración y diálogo intercultural}

El nexo entre migración e integración será un tema prioritario para la UE. La Comisión promoverá activamente la aplicación del Programa común para la integración sobre la base de los principios comunes sobre integración, abarcando todos los ámbitos de importancia incluidas las dimensiones laboral, socioeconómica, de salud pública, cultural y política. La Comisión desarrollará instrumentos que permitan una mayor participación de los diversos interesados, incluidos los emigrantes mismos, contribuyendo así a la promoción de una estrategia efectiva de integración. Ésta incluirá: a) el establecimiento de una plataforma de integración en la que los socios pertinentes puedan intercambiar puntos de vista con regularidad; b) la consolidación del papel desempeñado por las autoridades locales sobre la base de los resultados positivos de la Conferencia de Rotterdam "Integrando ciudades: políticas europeas y prácticas locales" de los días 9 y 10 de octubre; y c) la creación de un sitio Internet sobre temas de integración y nuevas ediciones del Manual de integración y del Informe anual sobre migración e integración.

La mejora de la integración en el mercado laboral es esencial. Se espera que el Grupo de alto nivel sobre integración social de las minorías étnicas y su pleno acceso al mercado laboral presente recomendaciones prácticas en 2007 que podrían enriquecer las actuales políticas. La UE debe centrar su atención a la educación de los niños en entornos de inmigrantes y debe utilizar el marco del Proceso de Educación y Formación 2010 con este fin. También es necesaria una mayor acción para garantizar que los emigrantes reciben una educación cívica sobre valores europeos fundamentales y aprenden la lengua de su país de acogida. Deben eliminarse de manera urgente las posibles barreras a la formación lingüística. Es igualmente importante la consolidación de la capacidad de la sociedad huésped para adaptarse a la diversidad. El Año Europeo de la Igualdad de Oportunidades para todos 2007 persigue aumentar la concienciación en torno a este problema.

Sería conveniente asimismo un diálogo intercultural como herramienta para la integración. El "Año europeo del diálogo intercultural 2008" dará prioridad al diálogo en la vida de cada día, por ejemplo, en las escuelas, en las actividades deportivas y culturales, y en el trabajo. Además, la UE debe continuar apoyando proyectos en el campo de la educación intercultural, la educación de los inmigrantes y la inclusión de la juventud desfavorecida a través de los programas pertinentes.

\subsection{Lucha contra la migración ilegal y la trata de seres humanos}

A nivel de la UE, hay que reforzar la lucha contra la inmigración ilegal en las áreas prioritarias identificadas en la Comunicación de la Comisión de 19 de julio de 2006. Las prioridades incluyen la necesidad de aumentar la confianza mutua y el intercambio de información entre los Estados miembros, incluida la regularización de los inmigrantes ilegales, la mejora del control del acceso al territorio de la UE y fijar unas sanciones para los patronos que ofrecen traba- 
jos a nacionales de terceros países que están en situación irregular. Aunque el fenómeno del trabajo no declarado no se limite a los emigrantes, la nueva legislación sobre sanciones contra los patronos de tales personas se propondrá en la primavera de 2007 dado que la oportunidad de obtener un trabajo en la UE sin la condición jurídica requerida es un factor que fomenta la inmigración ilegal. Paralelamente, se pondrá más énfasis en la aplicación de la legislación existente, que protegería asimismo a los emigrantes, en especial, las directivas sobre salud y seguridad en el lugar de trabajo. Además, los Estados deberían contar con apoyo para convertirse en Partes de los protocolos de la ONU en materia de tráfico ilícito y trata de seres humanos.

En 2007, EUROPOL dedicará especial atención a la lucha contra la inmigración ilegal facilitada y la trata de seres humanos y continuará desempeñando una función de inteligencia operativa y ofreciendo sesiones de formación y apoyo, que están abiertas a funcionarios de las fuerzas y cuerpos de seguridad de terceros países. Por su parte, FRONTEX debería, dentro del marco de la política de relaciones exteriores europea, establecer medidas técnicas de trabajo para operaciones conjuntas con los terceros países pertinentes, e invitarlos a participar en actividades operativas cuando convenga. Se están elaborando planes de acción sobre las diversas rutas migratorias, utilizando las recomendaciones de los funcionarios de enlace en materia de inmigración (ILO) a lo largo de las rutas. Las redes ILO deben reforzarse con el objetivo de tener por lo menos un funcionario de enlace en cada país africano clave de origen y tránsito. Además, deberían desarrollarse las condiciones para una "ILO de la UE", que podría actuar en nombre de varios Estados miembros.

El retorno y la readmisión seguirán siendo una parte fundamental de la gestión de la migración. Los Estados miembros deben contar con apoyo en el diseño y la aplicación de programas de retorno voluntario y planes para el regreso forzoso, incluidos vuelos conjuntos para el traslado. Constituye una prioridad apoyar a los Estados miembros a obtener los documentos necesarios para el retorno inmediato y la readmisión de los inmigrantes clandestinos. Se ha apoyado financieramente a los Estados miembros en sus esfuerzos para mejorar la gestión del retorno en todas sus dimensiones en el marco de las acciones preparatorias del RETORNO, cuyos resultados servirán para concentrar la programación plurianual del futuro Fondo Europeo para el Retorno. En este contexto, es igualmente importante que se adopte cuanto antes la Directiva propuesta por la que se establecen normas comunes a todos los Estados miembros sobre los procedimientos de retorno.

Se han concluido las negociaciones para un Acuerdo de readmisión CEUcrania al mismo tiempo que se han producido avances en las negociaciones con Marruecos. Se espera que se inicien pronto formalmente negociaciones similares con Moldava y Argelia. Como parte de un planteamiento equilibrado y basado en el diálogo sobre migración y visados previsto en los Planes de Acción de la PEV, deberían preverse negociaciones sobre readmisión y la facilitación de visados con cada país vecino una vez que se han resuelto las condiciones previas pertinentes. En términos de cooperación con los países ACP, la obligación de readmisión contenida en el artículo 13 Cotonú es decisiva, y es una base adecuada para los acuerdos bilaterales suplementarios de readmisión entre Estados miembros de la UE y los países ACP seleccionados. 
Su aplicación, sin embargo, debería considerarse en el marco más amplio del artículo 13.

La experiencia ha demostrado que, para alcanzar un acuerdo, la UE necesita ofrecer algo a cambio. En sus negociaciones bilaterales de readmisión, los Estados miembros están ofreciendo cada vez más otras formas de apoyo y ayuda a los terceros países para facilitar la celebración de tales acuerdos, y deben explorarse las posibilidades de aplicar este planteamiento más amplio a nivel de la UE.

La gestión integrada de las fronteras marítimas es una parte importante del planteamiento global y es el tema de una Comunicación separada, Reforzar la gestión de las fronteras maritimas meridionales de la Unión Europea.

\subsection{Asilo y protección a los refugiados}

Aunque es importante intensificar los esfuerzos para abordar los problemas de la migración ilegal, no se debe pasar por alto la necesidad de garantizar el acceso a los procedimientos de asilo para aquéllos que, en el contexto de los flujos migratorios, podrían necesitar la protección internacional. Las propuestas contenidas en el "Plan de acción de 10 puntos" del ACNUR presentado en la Conferencia de Rabat deben utilizarse para estimular la cooperación operativa. Es vital mantener los aspectos relativos al asilo y la protección como uno de los principales elementos de nuestra cooperación y diálogo con terceros países. Se debe continuar la ejecución de los programas regionales de protección, así como las actividades en otros ámbitos apoyando a los solicitantes de asilo y a las personas que necesitan protección internacional, tales como los originarios de Mauritania y África meridional. Se financiará asimismo un proyecto ejecutado por ACNUR para las actividades de protección en todos los países del sur y el este del Mediterráneo: Marruecos, Argelia, Túnez, Libia, Jordania, Siria y el Líbano.

\section{AYUDA FINANCIERA A LA POLÍTICA GLOBAL EUROPEA DE MIGRACIÓN}

\subsection{Apoyo a la dimensión exterior}

Según lo anunciado ya en el contexto de las conclusiones del Consejo Europeo de diciembre de 2005, la Comisión confirma su intención de intensificar su ayuda financiera en áreas relativas o relacionadas con la migración por lo que se refiere a sus relaciones con terceros países, incluyendo una asignación de hasta el $3 \%$ del IEVA y esfuerzos comparables por lo que se refiere a otros instrumentos financieros pertinentes. También se harán esfuerzos equivalentes en especial en el África subsahariana con objeto de abordar las causas profundas de la migración. La financiación se canalizará a través de programas geográficos establecidos conforme a los correspondientes instrumentos exteriores de ayuda y al programa temático sobre migración y asilo.

Por lo que se refiere al Fondo Europeo de Desarrollo (FED), la Iniciativa de Gobernanza de la UE para los países ACP es un mecanismo pertinente 
que permite a estos países obtener ayuda financiera adicional sustancial para desarrollar y poner en marcha reformas en materia de gobernanza. El acceso a estos recursos de incentivación del $10^{\circ} \mathrm{FED}$ dependerá del resultado del diálogo entre la Comisión y el país socio sobre los resultados anteriores y los compromisos futuros en el ámbito de la gobernanza, incluida la migración. Se pondrán a disposición otros recursos a través del programa UE sobre migración y desarrollo en África.

Además, tras la Conferencia de UE-África en Trípoli sobre Migración y Desarrollo, deberían explorarse las vías para prestar asistencia a aquellos países africanos septentrionales que desean ayudar a aliviar las presiones migratorias en los países subsaharianos a través de la ayuda financiera para coordinar sus esfuerzos con los de la UE.

El nuevo programa temático sobre migración y asilo se ha elaborado sobre la base de la experiencia adquirida con el programa Aeneas y de las lecciones aprendidas. Una innovación clave es que los recursos financieros se asignarán geográficamente, teniendo en cuenta el concepto de "ruta migratoria", o bien transversalmente, a través de iniciativas globales y multirregionales no exclusivamente ligadas a una única ruta migratoria.

Por último, el objetivo de la cooperación territorial europea de la política regional podría permitir también medidas que puedan ayudar a administrar las migraciones con los países vecinos, por ejemplo, mediante medidas de formación conjuntas para las patrullas fronterizas, las autoridades aduaneras y las fuerzas de policía, el refuerzo de los controles en los puertos y los aeropuertos, redes de intercambio de información sobre migración, el refuerzo de las capacidades y una legislación conveniente en materia de migración en los países de origen.

En el anexo $\mathbf{C}$, se recoge una información más específica relativa a las cantidades disponibles en los diversos instrumentos de financiación.

\subsection{Promover la solidaridad entre los Estados miembros}

La dotación de 4,020 millones asignada por la Autoridad Presupuestaria durante el período 2007-2013 al Programa marco titulado "Solidaridad y gestión de los flujos de migración" se divide en cuatro instrumentos financieros separados, cada uno de los cuales apoya diversos objetivos favorables de la gestión de los flujos de migración a la UE: el Fondo para las fronteras exteriores (1 820 millones de euros), el Fondo para el retorno (676 millones de euros), el Fondo europeo para los refugiados (699 millones de euros) y el Fondo para la integración ( 825 millones de euros). La parte fundamental de los recursos de cada uno de estos Fondos se pagará directamente a los Estados miembros en el marco de los dispositivos de gestión compartida. Estos nuevos instrumentos son específicos y complementarios de otra financiación comunitaria que persigue ocuparse de los hechos de la migración, incluidos los instrumentos de la Política Regional.

Los Estados miembros deberían adoptar un planteamiento estratégico a largo plazo sobre la utilización de estos recursos y apoyar las medidas nacionales que favorezcan la solidaridad entre los Estados miembros. Aunque 
este programa marco tiene por objeto principalmente reforzar la aplicación de la dimensión interior de las políticas comunitarias en materia de asilo, inmigración y control de las fronteras, buen número de las acciones impulsarán probablemente la consecución de los objetivos definidos en la presente Comunicación. Con el fin de poner de relieve esta relación, la Comisión propondrá unas directrices estratégicas con el fin de integrar los objetivos de la política en materia de migraciones en el marco operativo de cada uno de estos Fondos. La naturaleza global de estas acciones y la multiplicidad de sus objetivos se tendrán en cuenta y se reforzará la vocación de este programa marco para apoyar una serie de acciones vinculadas a la gestión de las migraciones en los Estados miembros.

La Comisión velará también para que las acciones comunitarias emprendidas en el marco de estos cuatro Fondos gestionados directamente por la Comisión, ofrezcan la posibilidad de ayudar a los Estados miembros a aplicar una política global en materia de inmigración gracias a la financiación de formas concretas de cooperación, la elaboración de herramientas y documentos de referencia comunes, proyectos piloto innovadores, y ofreciendo a las partes involucradas la posibilidad de contribuir con sus propios conocimientos y experiencia a la elaboración de las políticas comunitarias a través de investigaciones y actividades orientadas a las distintas políticas. La Comisión está asimismo comprometida en estudiar todas las posibilidades para generar un efecto palanca por medio de estos Fondos alcanzando acuerdos financieros con otras partes interesadas, como el Banco Europeo de Inversiones.

\section{CONCLUSIONES}

La Unión Europea debe adoptar una respuesta global a los desafíos cada vez mayores y en constante mutación planteados por la gestión de los flujos migratorios en un mundo globalizado. El año 2006 ha sido un año piloto para el Planteamiento global. La UE y sus Estados miembros consiguieron poner en marcha una cooperación concreta que no tenía precedentes. Sin embargo, el año 2006 habrá sido también un año de intensificación de la presión migratoria sobre la UE, particularmente en las fronteras del sur. Las acciones sobre este tema deben pues aumentarse y reforzarse en el marco de una perspectiva global, por ejemplo, mediante la aplicación de la "cláusula pasarela" prevista en el artículo 67 TCE con el fin de incrementar las capacidades de la UE para enfrentarse a los desafios ligados a la migración.

Desde 1999, la Unión Europea ha tomado una serie de iniciativas en favor de la instauración de una política común en materia de migraciones con el fin de crear una política de asilo común, de luchar contra la inmigración ilegal y la trata de seres humanos, de garantizar un tratamiento equitativo de los inmigrantes en situación regular, y establecer asociaciones con los países de origen y de tránsito. La Comisión está decidida a ir aún más lejos en esta vía, incluyendo propuestas legislativas al Consejo y al Parlamento acerca de la migración legal, y los derechos de los trabajadores emigrantes. Pero los Estados miembros deben también redoblar sus esfuerzos con el fin de garantizar una 
aplicación armoniosa de las medidas ya adoptadas de común acuerdo y completar la acción de la UE con sus propias iniciativas.

Es de la mayor importancia que se realice una evaluación de las acciones en curso con regularidad y se ajusten en consecuencia las estrategias de la UE, admitiendo que una respuesta duradera y conveniente al fenómeno de las migración exige unos esfuerzos constantes y de largo aliento así como importantes recursos.

\section{ANNEX A: REFERENCES}

The following list contains the full reference of each document quoted in the Communication, as well as some additional recent documents of relevance.

\subsection{COMMISSION COMMUNICATIONS}

- Communication from the Commission on Reinforcing the Management of the European Union's Southern Maritime Borders, COM(2006) of 30 November 2006.

- Communication from the Commission on Strengthening the European Neighbourhood Policy, COM(2006) 726 final of 29 November 2006.

- Communication from the Commission on The demographic future of Europe - from challenge to opportunity, $\mathrm{COM}(2006) 571$ of 12 October 2006.

- Communication from the Commission on Policy priorities in the fight against illegal immigration of third country nationals, COM (2006) 402 final of 19 July 2006.

- Communication from the Commission on Implementing the Hague Programme: the way forward, COM (2006) 331 final of 28 June 2006.

- Communication from the Commission on Promoting decent work for all: The EU contribution to the implementation of the decent work agenda in the world, COM(2006) 249 of 24 May 2006.

- Policy Plan on Legal Migration, COM(2005) 669 final of 21 December 2005.

- Communication from the Commission on an EU Strategy for Action on the Crisis in Human Resources for Health in Developing Countries, COM(2005) 642 of 12 December 2005.

- Communication from the Commission on Priority actions for responding to the challenges of migration: First follow-up to Hampton Court, $\operatorname{COM}(2005) 621$ final of 30 November 2005.

- Communication from the Commission on Migration and Development: Some concrete orientations, COM(2005) 390 final of 1 September 2005.

- Communication from the Commission on A Common Agenda for Integration: Framework for the Integration of Third-Country Nationals in the European Union, COM(2005) 389 final of 1 September 2005. 


\subsection{COUNCIL AND EUROPEAN COUNCIL CONCLUSIONS}

- Presidency Conclusions on the Global Approach to Migration: Priority actions focusing on Africa and the Mediterranean, European Council, Brussels, 15-16 December 2005.

- EU Strategy for Africa, European Council, SEC 15961/07, 15-16 December 2005.

- A Strategy for the External Dimension of JHA: Global Freedom, Security and Justice, 14366/3/05, 12 December 2005.

- Council Conclusions on A Common Agenda for Integration, 14390/05, 1-2 December 2005.

\subsection{OTHER DOCUMENTS}

- Annual Report on Migration and Integration SEC(2006) 892.

- National Action Plans for Social Inclusion 2006-2008.

- European Commission, DG Justice, Freedom and Security, Handbook on integration for policy makers and practitioners, November 2004, written by the Migration Policy Group, Brussels. The handbook can be downloaded from the following address: http://europa.eu.int/comm/ justice_home/doc_centre/immigration/integration/doc/handbook en.pdf

- Labour Migration Patterns in Europe: Recent Trends, Future Challenges, published in September 2006 in the series Economic Papers and available on the following website: http://ec.europa. eu/economy_finance/publications/economicpapers_en.htm).

\section{ANNEX B: OVERVIEW OF WORK CARRIED OUT ON EACH PRIORITY ACTION IN 2006}

The following gives details of work carried out on the priority actions during the course of 2006. Its purpose is to give an idea regarding the amount and type of work that has taken place, but is not intended to be comprehensive.

\subsection{INCREASING OPERATIONAL COOPERATION BETWEEN MEMBER STATES}

The FRONTEX Agency has coordinated several important joint operations to assist southern Member States. These activities have been a first test for the Agency and a show of solidarity with those Member States most affected by high numbers of migrants arriving every day. Among the important operations that have been carried out in the autumn are HERA II in the Canary Islands and off the coast of North-western Africa and NAUTILUS in the central Mediterranean. The purpose of these operations, which have also involved the cooperation of neighbouring African States, has been to enforce the control of the external maritime borders of the European Union, thereby disrupting and preventing illegal immigration by sea, and at the same time contribute 
to saving the lives of illegal immigrants in distress at sea (search and rescue operations). Moreover, FRONTEX has coordinated the detachment of experts in nationality identification from other Member States to Spain and Malta to assist the authorities of these two Member States in repatriating third country nationals who cannot legally remain in their territories.

Two important studies will inform future work. In July FRONTEX completed the feasibility study on a Mediterranean Coastal Patrols Network (MEDSEA). The study calls for the establishment of National Coordination Centres in the Member States, which would, twentyfour hours a day and seven days a week, coordinate the activities of the different national authorities involved, in cooperation with the national coordination centres of neighbouring Member States and third countries. FRONTEX is also carrying out the study on the technical feasibility of establishing a surveillance system covering the whole southern maritime borders of the EU and the Mediterranean Sea (BORTEC). This study will comprise an overview of the existing monitoring and surveillance systems in use, their area of coverage and their technical solutions, as well as the needs and wishes for further development with modern technology to cover the entire EU southern maritime borders. It is due to be completed by the end of 2006 .

The development of Regional Networks of Immigration Liaison Officers (ILOs) has progressed well as part of the overall Migration Routes Initiative. Four key migration routes from Africa to Europe were identified, and the presence of ILOs along these routes was determined. Reports on illegal immigration were drafted by these ILOs, which gave a picture of the situation in their respective countries and operational recommendations for stepping up cooperation along the routes. Meanwhile, Spain, France, Italy and the UK accepted leadership for each of the Regional Networks, and preparatory meetings got underway quickly. The Regional Networks will now work on developing an action plan for each route, consisting of concrete, operational projects that can be implemented to help combat illegal immigration.

In July the Commission put forward a proposal for the establishment of Rapid Border Intervention Teams to be managed by FRONTEX. Such teams would be made up of specially trained border guard officers of the national services of Member States who on short notice can be deployed to a requesting Member State to provide technical and operational assistance. All costs associated with the training and deployment of these officers by FRONTEX will according to the proposal be covered by the Community. The proposal is currently being discussed in the Council; quick adoption would mean they could become operational in the foreseeable future.

The Commission has also been carrying out an analysis of the law of the sea from the point of view of the fight against illegal immigration. The study examines Member State control powers in the different maritime spaces, as well as third countries' obligations on the basis of international sea law and maritime law and as regards the fight against the smuggling of migrants. The possibility for an institutionalised regional cooperation mechanism along the lines of the one existing amongst the Baltic States is also explored. 
EU Member States and the Commission played an active role in preparations for the UN High Level Dialogue on international migration and development. An EU Common Position was developed in the Council as a contribution to the Dialogue, supplemented by a Commission Communication. It set out the EU's approach to issues being covered by the Dialogue. The event itself was a success. High-level representatives from around the world joined together for two days to pledge their support for closer cooperation on migration and development issues. Many States expressed their wish to continue the dialogue through a global, informal and voluntary Forum. Belgium has offered to host the Global Forum on migration and development in July 2007. The aim will be to share expertise and best practice and enhance co-operation in the area of migration and development.

\section{PART I:}

\section{INCREASING OPERATIONAL COOPERATION BETWEEN MEMBER STATES}

\section{Call on FRONTEX to:}

1.1. implement border management measures in the Mediterranean region, in particular joint operations and pilot projects, as early as possible in 2006

Several joint operations and pilot projects were planned and implemented during 2006. The UK negotiated a framework partnership agreement with FRONTEX to allow UK officials to participate in joint operations on a case-by-case basis.

Project proposals were also submitted by Member States to the various Community programmes, including ARGO and AENEAS. These included the Spanish projects 'Seahorse', 'Atlantis' and 'Gate to Africa'.

Actions and events:

25 June-5 July

Operation Poseidon: this project measured the effectiveness of border control on the 'Balkan route'. Joint action was carried out to combat illegal immigration. It took place at various places along the land border between Greece and Turkey, as well as in harbours in Greece and Italy.

15 July-15 September

Operation AGIOS: a joint operation to tackle forged documents in Spain's Mediterranean ports. Deployment of EU officers started at the beginning of August. The implementation of the AGIOSProject is closely linked to the ARGO-funded project 'Gate of Africa'.

HERA I: 17 July-31 October

Operation HERA: a project that aimed to reduce the flow HERA II: 11 August to date of migrants from Senegal and Mauritania towards the Canary Islands. The objective was reached by raising the number of repatriations performed (HERA I - Identification and Return), as well as establishing patrols on the open sea near Senegal and Mauritania to reduce the departure of vessels from these shores (HERA II - Patrolling).

31 July-31 October Second phase is foreseen from mid-November to mid-December
Operation Migration Flow Malta: the main objective of this operation was to tackle the flow of illegal immigrants embarking from Libyan shores, to enhance knowledge and intelligence of the Maltese authorities and to increase the percentage of successfully identified illegal immigrants. 


\begin{tabular}{|c|c|}
\hline 5 October- 15 October & $\begin{array}{l}\text { Operation NAUTILUS: the main scope of this joint operation } \\
\text { was to patrol the area south of Sicily, Lampedusa and Malta } \\
\text { in the Mediterranean Sea to reduce the immigration flows } \\
\text { originating mainly from Libya. The involvement of Libya in } \\
\text { this project was sought. }\end{array}$ \\
\hline 1-22 November & $\begin{array}{l}\text { Operation Amazon: so-called Focal Point Offices were } \\
\text { established in international airports in Spain, Portugal, UK, } \\
\text { France, Italy, Netherlands and Germany, with the objective } \\
\text { of preventing illegal immigration. The focus was on Latin } \\
\text { American migration routes. }\end{array}$ \\
\hline Next steps: & - Continue carrying out joint operations. \\
\hline \multicolumn{2}{|c|}{ 1.2. Present a Risk Analysis report on Africa, building on recent studies, by May 2006.} \\
\hline \multicolumn{2}{|c|}{$\begin{array}{l}\text { FRONTEX shared its analysis report, Brief Assessment of Illegal Immigration Flows and } \\
\text { Routes on the African Continent, in May. This report assisted in the identification of the main } \\
\text { illegal immigration routes from Africa, and was used to support the work on setting up regional } \\
\text { ILO networks. }\end{array}$} \\
\hline \multicolumn{2}{|l|}{ Actions and events: } \\
\hline Spring & EUROPOL issued a study on the eastern Mediterranean. \\
\hline April & $\begin{array}{l}\text { FRONTEX delivered a tailored Risk Analysis report on } \\
\text { Illegal Immigration from Mauritania, identifying recently } \\
\text { used illegal immigration routes from Western Africa that } \\
\text { transit Mauritania. }\end{array}$ \\
\hline 30 May & $\begin{array}{l}\text { FRONTEX delivered the Risk Analysis on illegal migration } \\
\text { from Africa with special focus on Morocco and Libya and } \\
\text { presented the results to its Management Board Meeting in } \\
\text { Siofok. }\end{array}$ \\
\hline 22-23 May, Casablanca & Interpol meeting on illegal migration in Africa. \\
\hline 7-9 June, Brdo, Slovenia & $\begin{array}{l}\text { Joint ICMPD-EUROPOL meeting held within the framework } \\
\text { of the Dialogue on Mediterranean Transit Migration } \\
\text { (MTM), on the topic 'Migration Flows and Trends in the } \\
\text { Mediterranean'. }\end{array}$ \\
\hline 13-14 November, Porto & $\begin{array}{l}\text { Joint ICMPD-EUROPOL-FRONTEX meeting held within } \\
\text { the framework of the Dialogue on MTM, on the topic 'Project } \\
\text { Towards Comprehensive Response to Mixed Migration } \\
\text { Flows'. }\end{array}$ \\
\hline Next steps: & $\begin{array}{l}\text { - Risk analyses to be used in planning and preparation of } \\
\text { further operations. }\end{array}$ \\
\hline \multicolumn{2}{|c|}{$\begin{array}{l}\text { 1.3. Launch a feasibility study on reinforcing monitoring and surveillance of the southern } \\
\text { maritime border of the EU, namely in the Mediterranean Sea, and on a Mediterranean } \\
\text { Coastal Patrols Network involving EU Member States and North African countries, as } \\
\text { early as possible in } 2006\end{array}$} \\
\hline \multicolumn{2}{|c|}{$\begin{array}{l}\text { A support group currently consisting of } 14 \text { Member States was set up to undertake the } \\
\text { MEDSEA study, which was completed in July. The main recommendation of the study is to } \\
\text { establish national coordination centres in all Member States, which should on a } 24 / 7 \text { basis } \\
\text { coordinate the activities of Member States and third countries. }\end{array}$} \\
\hline
\end{tabular}




\begin{tabular}{|c|c|}
\hline \multicolumn{2}{|c|}{ Actions and events: } \\
\hline $1 \mathrm{March}$ & $\begin{array}{l}\text { A core team of experts from SP, IT, FR and GR started } \\
\text { work in FRONTEX. Participating Member States submitted } \\
\text { answers to a questionnaire circulated by FRONTEX. }\end{array}$ \\
\hline 6 April, Warsaw & First meeting of MEDSEA Support Group. \\
\hline 10 May, Warsaw & Second meeting of MEDSEA Support Group. \\
\hline 8 June, Helsinki & Third meeting of MEDSEA Support Group. \\
\hline 20 June, Warsaw & Fourth meeting of MEDSEA Support Group. \\
\hline 6 July, Warsaw & Fifth meeting of MEDSEA Support Group. \\
\hline 24 July & Study presented to the Council and the Commission. \\
\hline Next steps: & $\begin{array}{l}\text { Recommendations of the study to be implemented by } \\
\text { Member States as soon as possible, where necessary with } \\
\text { support from FRONTEX - timetable and costing to be put } \\
\text { together. } \\
\text { - Third countries to be contacted once the EU has a } \\
\text { clear view on how it wants to reinforce monitoring and } \\
\text { surveillance - sufficient flexibility for modifying the EU } \\
\text { approach to be kept to allow for the integration of the } \\
\text { wishes and views of third countries. }\end{array}$ \\
\hline
\end{tabular}

2. Explore the technical feasibility of establishing a surveillance system covering the whole southern maritime border of the EU and the Mediterranean Sea by the end of 2006. Such a system would use modern technology with the aim of saving lives at sea and tackling illegal immigration.

Working arrangements for the 'BORTEC' study were transferred from the Commission to FRONTEX.

\begin{tabular}{|l|l|}
\hline Actions and events: & $\begin{array}{l}\text { Expert group established to carry out the study, bringing } \\
\text { together both technical and operational people, including } \\
\text { those with expertise with the Galileo system. }\end{array}$ \\
\hline 5 April & $\begin{array}{l}\text { Coordination meeting between Commission and FRONTEX } \\
\text { to enable transfer and continuation of the work. }\end{array}$ \\
\hline 2 June & Project template approved. \\
\hline 7 July & First meeting of the Support Group. \\
\hline Next steps: & - Study due in December \\
\hline
\end{tabular}

3. Establish regional networks of Immigration Liaison Officers (ILOs) involving priority countries or regions as early as possible in 2006, and present reports on illegal immigration and trafficking, with the assistance where appropriate of ILOs in key countries, by May 2006.

The work to set up regional networks of ILOs has been closely linked to the migration routes initiative and the EU-Africa Ministerial Regional Conference held in Rabat. On the basis of existing data and statistics, four key migration routes from the African continent towards Europe were identified and selected for detailed work. For each of these routes the presence of 


\begin{abstract}
ILOs in the relevant countries was determined. The ILOs were approached to draft targeted illegal immigration reports and to submit concrete, operational recommendations for stepping up cooperation along the routes. EC Delegations in the relevant third countries identified immigration contact persons who supported the reporting activity. The ICONet system was adapted for specific use by the ILO Networks. In addition, for each key route a lead Member State was identified: SP, FR, IT and UK for regional networks 1 to 4 respectively. Leading Member States were requested to draft a six-month calendar of activities, including start-up regional meetings, with the final objective of developing an operational action plan for each route.
\end{abstract}

\title{
Actions and events:
}

\begin{tabular}{|c|c|}
\hline May-June & Illegal immigration reports drafted by ILOs. \\
\hline 14 June, Madrid & $\begin{array}{l}\text { Joint preparatory meeting for ILO regional networks } 1 \text { and } 2 \text {, } \\
\text { organised by SP and FR. }\end{array}$ \\
\hline $\begin{array}{l}6 \text { July, } \\
\text { Las Palmas de Gran Canaria }\end{array}$ & $\begin{array}{l}\text { Joint start up meeting for ILO regional networks } 1 \text { and } 2 \\
\text { organised by SP and FR, bringing together ILOs along both } \\
\text { routes. }\end{array}$ \\
\hline 6 October, London & $\begin{array}{l}\text { Joint preparatory meeting for ILO regional networks } 3 \text { and } 4 \\
\text { organised by IT and UK. }\end{array}$ \\
\hline 14-15 November, Rome & $\begin{array}{l}\text { Joint start up meeting for ILO regional networks } 3 \text { and } 4 \text {, } \\
\text { bringing together ILOs along both routes. }\end{array}$ \\
\hline Next steps: & $\begin{array}{l}\text { - Local meetings of ILO regional networks to be held } \\
\text { regularly } \\
\text { - Operational action plans to be drafted }\end{array}$ \\
\hline
\end{tabular}

4. Bring forward a proposal for the creation of rapid reaction teams made up of national experts able to provide rapid technical and operational assistance at times of high influxes of migrants, in accordance with the Hague Programme, by Spring 2006.

In July the Commission put forward a proposal for the establishment of Rapid Border Intervention Teams to be managed by FRONTEX. Such teams would be made up of specially trained border guardofficers of the national services of Member States who on short notice can be deployed to a requesting Member State to provide technical and operational assistance. All costs associated with the training and deployment of these officers by FRONTEX will, according to the proposal, be covered by the Community.

\section{Actions and events:}

\begin{tabular}{|l|l|}
\hline April & Study on legal competences of border guards completed. \\
\hline 19 July & $\begin{array}{l}\text { Proposal for a Regulation on the setting up of a mechanism for } \\
\text { the creation of Rapid Border Intervention Teams (RABITs) } \\
\text { submitted by the Commission to the European Parliament } \\
\text { and the Council. }\end{array}$ \\
\hline Next steps: & $\begin{array}{l}\text { - Council discussion of proposal, followed by adoption of } \\
\text { the Regulation } \\
\text { Continued work by Commission on a proposal for expert } \\
\text { intervention teams in the asylum context }\end{array}$ \\
\hline
\end{tabular}




\section{Ensure a substantial follow-up to the report of the Global Commission on International Migration, and prepare for the UN High Level Dialogue on Migration and Development that will be launched in September 2006}

Several preparatory events were organised in the lead-up to the HLD, and the Commission and Member States took an active part in all of them. A Commission Memorandum on EU policies, a Communication and an EU Common Position were all submitted as contributions to the preparations. The HLD itself was a success. High-level representatives from around the world committed to closer co-operation on migration and development issues and agreed to continue dialogue through a global forum.

\begin{tabular}{|c|c|}
\hline \multicolumn{2}{|l|}{ Actions and events: } \\
\hline 4-7 April, New York & $\begin{array}{l}\text { 39th Session of the Commission on Population and } \\
\text { Development. }\end{array}$ \\
\hline May & $\begin{array}{l}\text { Commission Memorandum on EU policies sent to the UN } \\
\text { Secretary-General by President Barroso. }\end{array}$ \\
\hline 12 June, Brussels & BE organised a seminar to discuss follow up to the HLD. \\
\hline 28-30 June, Turin & $\begin{array}{l}\text { International Symposium on Migration and Development } \\
\text { organised by DESA - Commission organised a session on } \\
\text { EU policies on migration and development, including as a } \\
\text { speaker a representative from the ACP Secretariat. }\end{array}$ \\
\hline 14 July & Commission Communication issued. \\
\hline 17 July, Brussels & $\begin{array}{l}\text { EU Common Position adopted by the General Affairs and } \\
\text { External Relations Council. }\end{array}$ \\
\hline 14-15 September, New York & $\begin{array}{l}\text { UN High Level Dialogue on International Migration and } \\
\text { Development. }\end{array}$ \\
\hline Next steps: & $\begin{array}{l}\text { - Participation in and support to the Global forum to be } \\
\text { hosted by BE in July } 2007\end{array}$ \\
\hline
\end{tabular}

6. Present an analysis of the existing international instruments on the law of the sea, including relevant aspects of refugee law, by March 2006.

The Commission has been carrying out this study with a particular focus on the Mediterranean. The study is essentially a gaps analysis identifying the issues that could be further explored. It is due to be published shortly.

Actions and events:

23-24 May, Madrid Meeting on Rescue at Sea and Maritime Interception in the Mediterranean, organised by UNHCR, attended by maritime and immigration representatives of almost all states bordering the Mediterranean, as well as other interested countries. The meeting illustrated the need for a concerted and complementary approach that involves all state actors, not only those engaged in rescue at sea and interception activities.

Three specific recommendations to come out of the meeting were: discussion on issues including rescue at sea, interception and disembarkation needs to continue; IMO Member States have a collective responsibility to comply with the SAR and SOLAS Conventions and the IMO guidelines; and with 


\begin{tabular}{|l|l|}
\hline & $\begin{array}{l}\text { improved data collection and strengthened cooperation, states } \\
\text { can continue to draw lessons from good practices identified } \\
\text { elsewhere and seek to benefit from the complementary } \\
\text { roles that may be played by IGOs in the area of reception, } \\
\text { screening and assistance in finding solutions for the various } \\
\text { categories of people. }\end{array}$ \\
\hline Next steps: & $\begin{array}{l}\text { - Commission to present report } \\
\text { Discussion of the analysis, and development of an EU } \\
\text { common position on the open legal questions }\end{array}$ \\
\hline
\end{tabular}

\subsection{DIALOGUE AND COOPERATION WITH AFRICA}

This first year has very much been a year of agenda-setting. With the recognition of the necessity to work in partnership with African and Mediterranean countries and to address the needs and concerns of all concerned, 2006 has acted as a preparatory year, paving the way for engagement with African states on the range of migration issues.

The amount of agenda space given to migration in regional fora this year has to some extent been surprising. An EU-Africa Ministerial Conference on Migration and Development was held in Tripoli on 22-23 November, which adopted a Joint Declaration. This was a highly significant event, with the EU and the whole of Africa coming together for the first time to make a political commitment to working together on migration. Similarly, other regional bodies gave their attention to migration - the ACP states held their first meeting of ministers responsible for asylum and migration, which resulted in a declaration and plan of action; migration was discussed and featured prominently in the conclusions of the EU-ECOWAS Troika; and for the first time migration was on the agenda as a discussion point at the ACPEU Council.

The ministerial conference held in Rabat in July was a successful and important event. A joint initiative between Morocco, Spain and France and co-financed by the Commission, the conference brought together West, Central and North African states with EU Member States to discuss common responses to migratory flows along the West African route. States committed themselves to developing a close partnership "to work together, in the framework of a global, balanced, pragmatic and operational approach, with respect for the fundamental rights and dignity of migrants and refugees, on the phenomenon of migratory routes". The conference agreed that this framework must involve countries of origin, transit and destination, and that central to this partnership is the fight against poverty and the promotion of sustainable development in African states. It will now be important to implement initiatives contained in the Action Plan, so as "to respond to the urgency of the situation and give visibility and credibility to the new dynamic brought about by the conference". The Rabat Declaration called for a second, follow up ministerial conference in two years' time at the latest.

Dialogue on the basis of article 13 of the Cotonou Agreement has been initiated with certain key Sub-Saharan African states. Meetings with the 
authorities in Mauritania, Senegal and Mali were extremely positive and productive, and discussion on migration now continues as part of the political dialogue led by Heads of Missions. Linked to this, migration is currently being incorporated into the programming exercise for the 10th European Development Fund (EDF). Where relevant, every Country Strategy Paper for ACP countries should contain a migration profile, and discussions between delegations, Member States and the third countries concerned should result in the identification of priorities for the funding of migration-related activities and projects for the next five years. Given the importance of assisting developing countries in managing migration, an intra-ACP migration facility of $€ 25$ million has been developed and programming work is underway. It will focus in particular on the management of south-south migration.

Mention should also be made of other ongoing work on the migration and development agenda. In March Belgium and the IOM, with the support of the World Bank and the Commission, organised a conference on migration and development. It successfully brought together high level representatives from across continents, to discuss achieving greater coherence between migration and development policies, forming partnerships among countries of origin, transit and destination, and involving migrant communities in the development of their home countries. In June the Commission hosted an expert meeting on migration and development. This was a good opportunity to share experiences and information on current projects, in particular regarding the four main topics: remittances, working with the diaspora, temporary and circular migration and brain drain. Participants agreed on the need to share information better and improve coordination in this field. Of course, this agenda links in with the UN High Level Dialogue, and it also needs to be reflected in EU assistance to interested countries in Africa.

With much of the political agenda-setting now in place, it will be important to move quickly to implement the commitments taken between Africa and the EU in the course of 2007 and beyond.

\section{PART II: DIALOGUE AND COOPERATION WITH AFRICA}

7. Work to make migration a shared priority for political dialogue between the EU and the African Union, including through regular senior officials' meetings to prepare for EU-Africa Ministerial Troika discussions.

Migration has featured prominently on the agenda of the AU and joint meetings between the $\mathrm{AU}$ and EU this year, with senior officials' meetings to prepare them as appropriate. Dialogue culminated in an EU-Africa Ministerial Conference on Migration and Development in Tripoli in November 2006.

Actions and events:

1 March, Addis Ababa

AU Commission and EU Commission Task Force - included discussion of the possibilities for a dialogue on migration.

3-5 April, Algiers

$\mathrm{AU}$ expert meeting - defined a draft common position on migration and development. 


\begin{tabular}{|c|c|}
\hline 8 May, Vienna & $\begin{array}{l}\text { EU-Africa Ministerial Troika, preceded by a senior officials' } \\
\text { meeting-discussion included the EU-pan African conference } \\
\text { on migration. }\end{array}$ \\
\hline 1-2 July, Banjul & $\begin{array}{l}\text { AU common position on migration and development } \\
\text { formally adopted at AU Summit. }\end{array}$ \\
\hline 11-12 September, Brussels & $\begin{array}{l}\text { EU-AU Commissions Joint Task Force-discussion focused } \\
\text { on exchange of information and preparation of the Tripoli } \\
\text { conference. }\end{array}$ \\
\hline 2 October, Addis Ababa & $\begin{array}{l}\text { Commission to Commission meeting involving many } \\
\text { Commissioners from both sides - migration was high on } \\
\text { the agenda and the principle of the Tripoli conference was } \\
\text { endorsed. }\end{array}$ \\
\hline 9 October, Brazzaville & $\begin{array}{l}\text { EU-Africa Ministerial Troika - concluded that the ministerial } \\
\text { conference on migration and development will take place in } \\
\text { Tripoli on } 22-23 \text { November. }\end{array}$ \\
\hline 22-23 November, Libya & $\begin{array}{l}\text { EU-Africa Ministerial Conference on Migration and } \\
\text { Development - joint declaration and trafficking action plan } \\
\text { adopted. Preparations tookplace in the High Level Working } \\
\text { Group on Asylum and Migration; an extended Troika } \\
\text { meeting hosted by Malta; and a senior official's meeting held } \\
\text { in Tripoli on } 21 \text { November. }\end{array}$ \\
\hline Next steps: & $\begin{array}{l}\text { - Follow-up to the Joint Declaration of the ministerial } \\
\text { conference. }\end{array}$ \\
\hline \multicolumn{2}{|c|}{$\begin{array}{l}\text { 8. Work in partnership with African countries and regional organisations, such as } \\
\text { ECOWAS, through a range of fora, initiatives and regional meetings, including an } \\
\text { EU-Africa Ministerial Conference in Morocco in } 2006 \text { and a conference on migration } \\
\text { and development in Brussels in March } 2006 .\end{array}$} \\
\hline \multicolumn{2}{|c|}{$\begin{array}{l}\text { Some successful high profile events have acted to keep migration firmly on the international } \\
\text { agenda this year and the subject of much political debate. }\end{array}$} \\
\hline \multicolumn{2}{|l|}{ Actions and events: } \\
\hline 6-8 February, Kenya & $\begin{array}{l}\text { IOM workshop on 'International Travel Documents and } \\
\text { Issuance Systems: Technical review of standards and systems } \\
\text { for East and Central African Governments, and participating } \\
\text { West African Governments. }\end{array}$ \\
\hline 15-16 March, Brussels & $\begin{array}{l}\text { Migration and Development Conference organised by } \\
\mathrm{BE} \text { and IOM, with the support of the World Bank and the } \\
\text { Commission. }\end{array}$ \\
\hline 4-6 April, Brussels & $\begin{array}{l}\text { African-European Inter-regional Dialogue on Managing } \\
\text { Labour Migration for Integration and Development, } \\
\text { organised by ILO with financial support from the EU. }\end{array}$ \\
\hline 13 April, Brussels & $\begin{array}{l}\text { First meeting of ACP Ministers in charge of Asylum, } \\
\text { Migration and Mobility - declaration and plan of action } \\
\text { adopted. }\end{array}$ \\
\hline 22-23 May, Vienna & $\begin{array}{l}\text { EU-ECOWAS Troika - migration was on the agenda and } \\
\text { figured prominently in the conclusions; agreement to set up a } \\
\text { joint working group on migration. }\end{array}$ \\
\hline
\end{tabular}




\begin{tabular}{|c|c|}
\hline 6 June, Lisbon & $\begin{array}{l}\text { IOM seminar on "Migration and development within the } \\
\text { Portuguese Speaking Countries Community - engaging } \\
\text { diasporas as agents for development". }\end{array}$ \\
\hline 6 June, Papua New Guinea & $\begin{array}{l}\text { ACP-EU Council - migration was on the agenda as a ' } \mathrm{C} \text { ' } \\
\text { point (discussion) for the first time. }\end{array}$ \\
\hline 28-29 June, Niamey & $\begin{array}{l}\text { Technical seminar, funded by the AENEAS } 2004 \text { Across } \\
\text { Sahara project - focused on the situation in Libya and Niger } \\
\text { concerning a range of issues such as illegal immigration, } \\
\text { transit migration, visas, to readmission and return, asylum } \\
\text { and statistics, and included an analysis report drawn up on } \\
\text { the basis of questionnaire replies from the Nigerian and } \\
\text { Libyan authorities. }\end{array}$ \\
\hline 10-11 July, Rabat & $\begin{array}{l}\text { Euro-Africa ministerial conference on migration and } \\
\text { development - declaration and action plan adopted, with the } \\
\text { commitment to hold a next ministerial within two years to } \\
\text { assess progress. Preparation took place in steering committee } \\
\text { meetings, senior officials' meetings (including one hosted by } \\
\text { Senegal), and in the High Level Working Group on Asylum } \\
\text { and Migration with the participation of the Ambassador of } \\
\text { Morocco. }\end{array}$ \\
\hline 26-27 October, Niamey & $\begin{array}{l}\text { EU-ECOWAS Troika - migration was once again high on } \\
\text { the agenda and in the conclusions. }\end{array}$ \\
\hline Next steps: & $\begin{array}{l}\text { - Ongoing follow-up and implementation of initiatives } \\
\text { contained in the Rabat action plan } \\
\text { - Develop East Africa migration route initiative } \\
\text { - Rapidly set up EU-ECOWAS joint working group on } \\
\text { migration and initiate its work }\end{array}$ \\
\hline
\end{tabular}

9. Explore the feasibility of a migration routes initiative for operational cooperation between countries of origin, transit and destination, with a view to developing a concrete initiative in $\mathbf{2 0 0 6 .}$

Development of the migration route concept has made good headway, with several steps being taken to bring together countries of origin, transit and destination along the same migratory route. Four key migration routes on the African continent towards Europe were identified, as set out in action 3 above.

Other related initiatives include a General Action Plan prepared by EUROPOL concerning cooperation with third countries for the purposes of Police Chiefs Task Force; and a pilot project proposal submitted by BE to Morocco on return and reintegration of Malians, as well as a regional initiative to help Mali dismantle smuggling networks.

\section{Actions and events:}

10-11 July, Rabat

Euro-Africa ministerial conference on migration and development, which aimed at identifying operational action along the West Africa migration routes.

7-10 November, Las Palmas

SP hosted a police conference in the framework of the Seahorse project, which was attended by Member States, FRONTEX and EUROPOL. 


\begin{tabular}{|l|l|}
\hline July & $\begin{array}{l}\text { UK presented proposals for an East Africa Migration Routes } \\
\text { Initiative to the High Level Working Group on Asylum and } \\
\text { Migration. This includes a stock-take of Member States' } \\
\text { activities in the region; an analysis of the gaps in relevant } \\
\text { transit countries and the requirements; and the ILO regional } \\
\text { network developments with Italy. }\end{array}$ \\
\hline Next steps: & $\begin{array}{l}\text { A global approach action plan for each of the routes will } \\
\text { be developed, using a variety of sources including the ILO } \\
\text { reports. }\end{array}$ \\
\hline
\end{tabular}

10. Enhance dialogue by spring 2006 with key sub-Saharan African states on the basis of Article 13 of the Cotonou Agreement, covering a broad range of issues from institution and capacity building and effective integration of legal migrants to return and the effective implementation of readmission obligations, in order to establish a mutually beneficial cooperation in this field.

The Commission proposed launching bilateral dialogue on migration on the basis of article 13 of the Cotonou Agreement between the EU and Senegal, Mali, Cameroon, Ghana, Nigeria, Mauritania and Niger. This has so far proceeded with Mauritania, Senegal and Mali. Three other countries had to be removed from the list due to varying political circumstances. Seven Member States proposed adding Ethiopia, Sudan, Eritrea and Somalia to this initial list of countries; BE proposed adding Guinea (Conakry) and Rwanda; and DE proposed adding Burkina Faso.

At the same time migration is being integrated into the Country Strategy Papers for ACP countries, as part of the programming exercise for the 10th European Development Fund (EDF). Once drafted by Delegations and ACP countries, with the participation of Member States, they will be formally adopted by the EDF-Committee.

Actions and events:

\begin{tabular}{|c|c|}
\hline January & $\begin{array}{l}\text { Commission delegations in selected countries were contacted } \\
\text { to explore the possibilities of article } 13 \text { Cotonou dialogue. }\end{array}$ \\
\hline April-June & $\begin{array}{l}\text { Technical mission Mauritania in the framework of the Rapid } \\
\text { Reaction Mechanism; the final decision on financial support } \\
\text { was taken in June to a total of } 2.45 \text { million euro. }\end{array}$ \\
\hline 5-6 June, Dakar & $\begin{array}{l}\text { Informal contacts were made with several African countries } \\
\text { in the margins of the meeting in Dakar that prepared the Rabat } \\
\text { Ministerial Conference on migration and development. }\end{array}$ \\
\hline 23-25 May & $\begin{array}{l}\text { Commission mission to Mauritania and Senegal to initiate } \\
\text { article } 13 \text { dialogue, so that it can continue at Head of Mission } \\
\text { level in the context of the regular political dialogue (article } \\
8 \text { Cotonou). }\end{array}$ \\
\hline 25-28 September & Commission mission to Mali in context of article 13 . \\
\hline September-November & $\begin{array}{l}\text { Technical mission to Senegal in the framework of the Rapid } \\
\text { Reaction Mechanism; the financial decision will be taken } \\
\text { shortly. }\end{array}$ \\
\hline 18-20 October, Brussels & $\begin{array}{l}\text { Training workshop for EC delegations on migration in EC } \\
\text { external assistance. }\end{array}$ \\
\hline
\end{tabular}




\begin{tabular}{|c|c|}
\hline Next steps: & $\begin{array}{l}\text { - Heads of Mission in Ethiopia, Sudan, Eritrea and Somalia } \\
\text { to assess the feasibility of launching dialogue with these } \\
\text { countries. } \\
\text { - Article } 13 \text { dialogue to be initiated with further key } \\
\text { countries, with regular reporting back to Member States, } \\
\text { and to be actively continued with all relevant countries. }\end{array}$ \\
\hline \multicolumn{2}{|c|}{$\begin{array}{l}\text { 11. Establish and implement a pilot Regional Protection Programme (RPP) involving } \\
\text { Tanzania as early as possible in } 2006 \text {, with a steering group to oversee the programme. } \\
\text { Based on findings from the pilot, develop plans for further programmes in Africa }\end{array}$} \\
\hline \multicolumn{2}{|c|}{$\begin{array}{l}\text { Following the Council Conclusions on Regional Protection Programmes in November } 2005 \text {, } \\
\text { several proposals for projects to initiate the pilot RPPS were submitted under the } 2005 \text { AENEAS } \\
\text { call for proposals. One of these was submitted by UNHCR and focused specifically on } \\
\text { Tanzania and the surrounding region. The proposal was selected by the AENEAS Management } \\
\text { Committee, and is expected to get underway in } 2007 \text {. The project will include strengthening } \\
\text { the capacity of national authorities to protect refugees, improving security in refugee camps, } \\
\text { promoting voluntary return of Burundian refugees, enhancing access to resettlement, and } \\
\text { registration of refugees. }\end{array}$} \\
\hline \multicolumn{2}{|l|}{ Actions and events: } \\
\hline 14 February, Brussels & $\begin{array}{l}\text { Expert meeting with Member States convened by the } \\
\text { Commission. }\end{array}$ \\
\hline July & AENEAS project proposal for Tanzania accepted. \\
\hline Next steps: & $\begin{array}{l}\text { - Initiate projects as soon as contracts signed. } \\
\text { - Initiate UNHCR-run project for North Africa. } \\
\text { - Develop further protection-oriented projects with } \\
\text { UNHCR. }\end{array}$ \\
\hline
\end{tabular}

12. Carry out a study to improve understanding of the root causes of migration to underpin the longterm approach

The Joint Research Centre is currently carrying out a case study focusing on Senegal and Mali. It will analyse the root causes of migration in these two countries and their policy implications.

\begin{tabular}{|l|l|}
\hline \multicolumn{2}{|l|}{ Actions and events: } \\
\hline July & $\begin{array}{l}\text { Drafting commenced and Member States were invited to } \\
\text { provide information on existing studies on root causes. }\end{array}$ \\
\hline Next steps: & $\begin{array}{l}\text { - Study expected in November 2006. } \\
\text { - Study should be discussed and used as part of the long- } \\
\text { term EU approach. }\end{array}$ \\
\hline
\end{tabular}

13. Develop regular dialogue with UNHCR as early as possible in 2006, to share experience and expertise on working with countries in Africa.

Dialogue with UNHCR on the implementation continued via regular meetings.

\begin{tabular}{|l|l|}
\hline Next steps: & $\begin{array}{l}\text { A high-level meeting will be organised between the } \\
\text { Commission and UNHCR in early January in order to } \\
\text { operationalise the UNHCR 10-Point Plan of Action } \\
\text { presented at the Rabat conference; the feasibility of } \\
\text { holding such meetings regularly to update on the situation } \\
\text { in different countries will be examined. }\end{array}$ \\
\hline
\end{tabular}


14. Launch initiatives in early 2006 to promote cheaper and more easily available remittance services, and support ongoing efforts by international organisations to improve data on remittance flows; consider supporting efforts of African states to facilitate members of diasporas to contribute to their home countries, including through co-development actions, and explore options to mitigate the impact of skill losses in vulnerable sectors

This wide agenda was the subject of much discussion during the year. An expert meeting organised by the Commission in June was a useful opportunity to share experiences and best practice, while productive discussion took place in the UN High Level Dialogue on Migration and Development in New York and the Africa-EU Ministerial Conference on Migration and Development in Libya.

Actions and events:

15-16 March, Brussels $\quad$ Migration and Development Conference organised by $\mathrm{BE}$ and IOM, with the support of the World Bank and the Commission.

20 June, Brussels $\quad$ Member State expert meeting on the main subjects covered by the September 2005 Migration and Development Communication convened by the Commission.

10-11 July, Rabat $\quad$ Euro-Africa ministerial conference on migration and development.

14-15 September, New York UN High Level Dialogue on Migration and Development.

13-14 November, London $\quad$ UK-World Bank Conference on Remittances - UK also made available its publication on the UK remittances market.

22-23 November, Tripoli $\quad$ EU-Africa Ministerial Conference on Migration and Development.

Next steps:

- Establish 'virtual' working groups on each of the main topics on the migration and development agenda, involving Member States, international organisations, Commission services and other experts as appropriate.

- Support projects on migration and development in interested African countries as part of EU financial assistance under the EDF, the MEDA programme, Aeneas or the future thematic programme on migration.

15. Establish information campaigns targeting potential migrants to highlight the risks associated with illegal migration and raise awareness about legal channels for migration.

IOM carried out information campaign work with Senegal during the summer.

Portugal has also organised information campaigns in embarkation/disembarkation bulletins disseminated at airports of origin and destination and travel agencies, with information concerning the requirements for legal entry in Portugal and raising awareness of the risks of illegal migration and networks of trafficking in human beings. 


\subsection{WORK WITH NEIGHBOURING COUNTRIES}

Although progress in the EuroMed framework has not developed as quickly as was hoped, important and productive senior officials' meetings were held in June and October. These renewed motivation and optimism for furthering cooperation on migration, which has been given concrete expression through the preparation of a working document detailing projects and best practices of each of the EuroMed partners relating to legal migration, migration and development and the fight against illegal migration, and a more detailed action programme with recommendations for future cooperation. Portugal has also offered to host a EuroMed Ministerial meeting on migration in 2007.

Meanwhile, work with individual North African countries has made good headway. The dialogue and cooperation with Morocco has intensified and the EU has decided to grant political and considerable financial support to Morocco (up to $€ 90$ million) to assist this country in its efforts to better manage migration. A major step forward was taken with a twinning project on the fight against illegal migration, as well as budgetary support for purchasing border control equipment. Negotiations on an EC Readmission agreement have also continued, and are expected to be launched with Algeria soon. An important programme of support ( $€ 10$ million) to the Algerian border police, approved in 2005, is ongoing. Discussions with Libya on issues concerning migration have also continued and Libya has invited a team of EU experts to carry out a mission to its southern borders. All in all, cooperation with the Mediterranean countries is set to develop strongly in the future, on the basis of the relevant Association Agreements and Neighbourhood Policy Action Plans.

\section{PART III: WORK WITH NEIGHBOURING COUNTRIES}

\section{Hold a EuroMed Ministerial meeting on migration in 2006}

At a positive senior officials' meeting, it was agreed to collect information on projects and best practice in areas identified by the Barcelona Summit in November 2005: legal migration, migration and development and illegal migration, trafficking and return issues. With contributions from all 35 delegations, the Commission undertook to prepare a background document with all the information provided, as well as a more detailed action programme containing recommendations for future cooperation.

Actions and events:

\begin{tabular}{|l|l|}
\hline 20 June, Brussels & $\begin{array}{l}\text { Senior officials' meeting to discuss further cooperation and } \\
\text { to prepare the Ministerial meeting.. }\end{array}$ \\
\hline 20 October, Brussels & $\begin{array}{l}\text { Second senior officials' meeting, which discussed the draft } \\
\text { action programme. }\end{array}$ \\
\hline Next steps: & $\begin{array}{l}\text { - Continue developing ideas for concrete joint projects. } \\
\text { Continue preparations for the EuroMed Ministerial, } \\
\text { planned to take place during the Portuguese Presidency in } \\
2007 .\end{array}$ \\
\hline
\end{tabular}


17. Engage Mediterranean third countries in the feasibility study of a Mediterranean Coastal Patrols Network, Mediterranean surveillance system and related pilot projects, where appropriate.

See priority action 1.3 .

18. Make available experiences and best practices where appropriate from other regional cooperation structures, including those relating to the Baltic Sea.

9 June, Helsinki

Finland hosted a Member State expert visit to the Helsinki Headquarters of the Border Guards to assess the Baltic experience in dealing with Border security management, comprising technical means, legal framework, regional cooperation and operational activities.

19. Use all available frameworks for cooperation with Mediterranean partners, including those mentioned below, to prevent and combat illegal migration and trafficking in human beings, build capacity to better manage migration, and explore how best to share information on legal migration and labour market opportunities, for example through the development of migration profiles and through strengthening subregional fora.

The possibilities of implementing the priority actions in the framework of the $5+5$ cooperation were explored in the $5+5$ group under the Chair of France and then Spain. For example, France, Spain and Morocco are working on a joint project that aims to promote the institutional capacity between these countries on labour migration, so as to connect labour demand and offer between them. The project started in December 2005 and will finish in November 2008.

Meanwhile, the concept of the Migration Profile has been developed. The IOM and the JRC both drafted initial proposals for how the tool could work. The idea was then formally presented at the UN High Level Dialogue in New York on 14-15 September. Funding has been earmarked in the AENEAS 2006 call for proposals to further develop Migration Profiles for key countries.

\begin{tabular}{|c|c|}
\hline 12 May, Nice & $\begin{array}{l}5+5 \text { Interior Ministers }- \text { Commission was invited to take part } \\
\text { in the lunch. }\end{array}$ \\
\hline 29-30 June, Paris & $\begin{array}{l}\text { Follow-up meeting to the ministerial conference on migration } \\
\text { held in Paris in November } 2005 \text {, with discussion including } \\
\text { migration and development, labour migration and reception } \\
\text { and integration of migrants. }\end{array}$ \\
\hline Next steps: & $\begin{array}{l}\text { - Continue using all relevant fora to further work on the } \\
\text { Global Approach. } \\
\text { - Continue developing the Migration Profile concept, in } \\
\text { particular using AENEAS funding. }\end{array}$ \\
\hline
\end{tabular}

\section{Undertake priority work with the following three countries:}

20.1 Morocco - implement projects to combat trafficking and conclude negotiations of the ECMorocco readmission agreement as early as possible.

Implementation of various projects using MEDA and AENEAS funding has been promoted, and negotiations on an EC Readmission agreement have continued. Morocco was also host of the Euro-Africa ministerial conference on migration and development, held in Rabat in July.

Actions and events: 


\begin{tabular}{|l|l|}
\hline 18 May & EU-Morocco subcommittee on JHA. \\
\hline $10-11$ July, Rabat & $\begin{array}{l}\text { Euro-Africa ministerial conference on migration and } \\
\text { development. }\end{array}$ \\
\hline 22 November, Rabat & $\begin{array}{l}\text { Meeting of the EU-Morocco subcommittee on migration and } \\
\text { social affairs and readmission discussions. }\end{array}$ \\
\hline November, Rabat & $\begin{array}{l}\text { EU-Morocco Working Group on migration and social } \\
\text { affairs. }\end{array}$ \\
\hline Next steps: & $\begin{array}{l}\text { Continuation of the negotiations on an EC Readmission } \\
\text { agreement. }\end{array}$ \\
Effective implementation of cooperation projects and \\
continued dialogue on migration-related issues.
\end{tabular}

20.2. Algeria - hold a first meeting in early 2006 to take forward cooperation on the basis of the migration provisions of the EC-Algeria Association Agreement and begin the negotiation of the readmission agreement as quickly as possible on the basis of the mandate given to the Commission.

Algeria hosted an AU expert meeting on migration and development in April. Negotiations on the EC readmission agreement are expected to be launched in early 2007.

\section{Actions and events:}

\begin{tabular}{|c|c|}
\hline $16 \mathrm{May}$ & First EU-Algeria Association Council meeting. \\
\hline Next steps: & $\begin{array}{l}\text { - First meeting of the EU-Algeria subcommittee on } \\
\text { migration and social affairs, 5-6 December, Algiers. } \\
\text { - Effective start of negotiations on an EU-Algeria } \\
\text { readmission agreement. } \\
\text { - Effective dialogue on migration-related issues. }\end{array}$ \\
\hline
\end{tabular}

20.3 Libya - conclude the work to agree the EU-Libya Action Plan on migration as early as possible in 2006, in accordance with the Council Conclusions of 3 June 2005 on initiating dialogue and cooperation with Libya on migration issues, and implement projects as soon as possible thereafter.

The Commission has been doing its utmost to work bilaterally with Libya in addressing the issue of illegal migration, and there have been various missions to discuss cooperation. Libya hosted the EUAfrica Ministerial Conference on Migration and Development in November. The AENEAS-funded IOM Programme for the Enhancement of Transit and Irregular Migration Management in Libya (TRIM) has continued.

Actions and events:

\begin{tabular}{|l|l|}
\hline February & $\begin{array}{l}\text { Commission visited Tripoli and presented proposals for } \\
\text { concrete cooperation and dialogue on migration issues to the } \\
\text { Libyan side. }\end{array}$ \\
\hline 21-22 May & $\begin{array}{l}\text { Commission visited Tripoli. Libya confirmed interest in } \\
\text { developing dialogue and cooperation with the EU, although } \\
\text { not in the framework of the Barcelona process. }\end{array}$ \\
\hline May & $\begin{array}{l}\text { Response received from Libya, expressing willingness to } \\
\text { host the AUEU conference and inviting an expert mission to } \\
\text { the southern border. }\end{array}$ \\
\hline
\end{tabular}




\begin{tabular}{|l|l|}
\hline 4-6 July & $\begin{array}{l}\text { Commission visited Libya to discuss enhanced cooperation } \\
\text { in several areas including migration. }\end{array}$ \\
\hline Next steps: & $\begin{array}{l}\text { - Organise expert mission to Libya's southern borders as a } \\
\text { matter of priority. } \\
\text { - Explore possibilities for developing an EU-Libya joint } \\
\text { risk analysis. } \\
\text { - Examine the possibility of establishing direct contacts } \\
\text { between FRONTEX and Libya to explore areas for } \\
\text { joint cooperation, for instance in carrying out joint risks } \\
\text { assessment and joint patrolling. }\end{array}$ \\
\hline
\end{tabular}

21. Intensify research to improve understanding and management of migratory flows, building on the migration component of the regional JHA I MEDA programme.

Activities launched by the Consortium for Applied Research on International Migration in the Mediterranean region (CARIM), hosted by the European University Institute in Florence, Italy, were continued throughout the year.

\section{Actions and events:}

\begin{tabular}{|c|c|}
\hline May & $\begin{array}{l}\text { Financed by AENEAS 2004, a new website, Migration de } \\
\text { retour vers le Maghreb (MIREM), was opened at http:// } \\
\text { www.mirem.eu. It is hosted by the European University } \\
\text { Institute as part of CARIM. The MIREM project aims to } \\
\text { examine the current challenges linked to return migration } \\
\text { and its impact on development in Maghreb countries. The } \\
\text { website includes data and information on the premises and } \\
\text { implications of the EU common return policy; patterns of } \\
\text { cooperation on readmission and their challenges; and the } \\
\text { bilateral agreements linked to readmission involving the } \\
\text { Maghreb countries and EU Member States. There is also an } \\
\text { annotated inventory of the statistical data on return migration } \\
\text { to the Maghreb countries, including various typologies of } \\
\text { returnees, and studies related to the return mechanisms that } \\
\text { have been implemented in the Maghreb. }\end{array}$ \\
\hline 20 June, Brussels & $\begin{array}{l}\text { Commission's proposals for JHA MEDA II were presented } \\
\text { to the EuroMed partners at the senior officials' meeting on } \\
\text { migration. }\end{array}$ \\
\hline Next steps: & $\begin{array}{l}\text { CARIM will continue its research on migration in the } \\
\text { Maghreb region. } \\
\text { - Inspired by the success of the academic network } \\
\text { (CARIM) linking migration research institutes of all } \\
\text { the Mediterranean countries and funded by the MEDA } \\
\text { programme, the EU should facilitate an academic } \\
\text { conference in view of the establishment of a pan-African } \\
\text { network of migration 'observatories' and/or migration } \\
\text { research institutes. }\end{array}$ \\
\hline
\end{tabular}

22. Help strengthen links between North and sub-Saharan African countries in the framework of the possible migration routes initiative.

See priority action 9 


\section{Continue dialogue and cooperation with UNHCR in helping third countries develop capacity for refugee protection.}

The Commission has financed a UNHCR project aimed at building asylum capacity in North Africa. The results of the project have been just transmitted to the Commission.

In order to ensure continuity and build on this first experience, the Commission intends to finance under AENEAS 2006 another UNHCR project for protection actions in all the Southern and Eastern Mediterranean countries, from Morocco to Syria.

\section{Hold a conference on The role of internal security in relations between the EU and its neighbours in Vienna in May 2006.}

Following adoption by the Council of the Strategy for the External Dimension of the Area of Freedom, Security and Justice in December 2005, the Vienna Ministerial Conference was held on 4-5 May. The 'Vienna Declaration on Security Partnership' was adopted, and then welcomed by the European Council of 15-16 June.

\section{Actions and events:}

\begin{tabular}{|l|l|}
\hline 4-5 May, Vienna & Conference took place. \\
\hline Next steps: & $\begin{array}{l}\text { - The Commission will continue to monitor progress of the } \\
\text { Strategy and will report to the Council every } 18 \text { months. }\end{array}$ \\
\hline
\end{tabular}

\section{ANNEX C: FINANCING}

In the context of its December 2005 Conclusions on priority actions focusing on Africa and the Mediterranean, the European Council welcomed the increased priority being given to migration and the Commission's intention to intensify its financial assistance in areas concerning or related to migration in respect of its relations with third countries, including by an allocation of up to $3 \%$ of the ENPI, and comparable efforts in respect of other relevant financial instruments. It also called for equivalent efforts in particular in Sub-Saharan Africa, with a view to tackling the root causes of migration.

As stated in section 4.1. of this Communication, funding will be channelled through the geographic programmes established under the relevant external assistance instruments and theThematic programme on migration and asylum.

As regards the European neighbourhood countries, the $3 \%$ commitment represents an amount of about $€ 50$ million a year, of which $€ 30$ million will be brought by the ENPI contribution to the Thematic programme on migration and asylum. The remaining part will be financed under the national, regional and cross-border geographic programmes established within the ENPI.

In addition to the initiatives on migration and asylum financed under the geographic programmes of the DCI, the DCI will contribute for about $€ 25$ million a year to the Thematic programme on migration and asylum, for funding projects in developing countries located outside of the European Neighbourhood.

As regards the ACP countries, and within the framework of the $9^{\text {th }} \mathrm{EDF}$, an overall $€ 40$ million has been set aside for the EU Programme on Migration and Development in Africa, including the $€ 25$ million intra-ACP migration 
facility, whose specific destination is currently in an identification phase. Concerning the $10^{\text {th }} \mathrm{EDF}$, which covers the period 2008-13, migration is being fully incorporated into the programming of Country and Regional Strategy Papers. Through the EU Governance Initiative and its 'incentive tranche', ACP countries will be able to obtain substantial additional financial support to develop and implement governance reforms. Access to these $10^{\text {th }} \mathrm{EDF}$ incentive resources will depend on the outcome of a dialogue between the Commission and the partner country on the past performance and future commitments in the area of governance, including on migration.

Finally, it should not be overlooked that further to the geographic programmes and to the thematic programme on asylum and migration, other thematic programmes / instruments such as the European Instrument for Democracy and Human Rights (EIDHR), the Thematic programme for cooperation with non-state actors and local authorities or the Thematic programme "investing in people" can also occasionally provide funds on specific migration relevant issues such as trafficking in human beings or on aspects of the migration and development debate. The new Structural Funds' regulations make possible that in the context of cross-border, transnational and interregional cooperation, the ERDF may finance expenditure incurred in implementing operations or parts of operations on the territory of countries outside the European Community (up to a limit of $10 \%$ of the amount of its contribution to the operational programme concerned). 